\title{
DNA Repair Gene Alterations and PARP Inhibitor Response in Patients With Metastatic Castration-Resistant Prostate Cancer
}

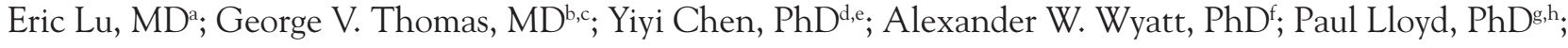 \\ Jack Youngren, $\mathrm{PhD}^{\mathrm{g}, \mathrm{h}}$; David Quigley, $\mathrm{PhD}{ }^{\mathrm{g}, \mathrm{i}}$; Raymond Bergan, MD, ; Shawna Bailey, $\mathrm{MPH}^{\mathrm{c}}$; \\ Tomasz M. Beer, MD ${ }^{\mathrm{a}, \mathrm{c}}$; Felix Y. Feng, MD ${ }^{\mathrm{ghh,j}}$; Eric J. Small, MD ${ }^{\mathrm{g}, \mathrm{h}}$; and Joshi J. Alumkal, MD ${ }^{\mathrm{a}, \mathrm{c}}$
}

\begin{abstract}
Background: PARP inhibition is a promising therapeutic strategy for the treatment of men with metastatic castration-resistant prostate cancer whose tumors harbor homologous recombination DNA repair gene alterations. However, questions remain for many practicing clinicians about which patients are ideally suited for PARP inhibitor treatment. This report details our institutional experience using PARP inhibitor therapy in patients whose tumors harbored specific DNA repair gene alterations. Patients and Methods: We performed a retrospective chart review to identify patients at Oregon Health \& Science University who were treated with PARP inhibition. We identified 8 patients and determined the impact of the specific DNA repair gene alterations on tumor response and time on treatment with PARP inhibition. Results: A number of DNA repair gene alterations were identified. Three patients had pathogenic $B R C A 2$ mutations and one had a $B R C A 2$ mutation of uncertain significance. Conversely, the 4 other patients' tumors harbored alterations in other DNA repair genes, none of which were clearly pathogenic. A statistically significant difference in benefit was seen between patients whose tumors harbored $B R C A 2$ gene alterations and those whose tumors did not, as measured by $>50 \%$ decline in prostate-specific antigen levels ( $100 \%$ vs $0 \%$; $P=.03$ ) and duration on therapy ( 31.4 vs 6.4 weeks; $P=.03$ ). Conclusions: Our results demonstrate that not all DNA repair alterations are equally predictive of PARP inhibitor response. Importantly, all responding patients had tumors harboring BRCA2 DNA repair alterations, including one without a known pathogenic mutation. Conversely, among the 4 nonresponders, several DNA repair alterations in genes other than $B R C A 2$ were identified that were not clearly pathogenic. This demonstrates the need to carefully examine the functional relevance of the DNA repair alterations identified, especially in genes other than BRCA2, when considering patients for PARP inhibitor treatment.
\end{abstract}

${ }^{\text {aDepartment }}$ of Medicine, Oregon Health \& Science University, Portland, Oregon; ${ }^{b}$ Department of Pathology \& Laboratory Medicine, Knight Cancer Institute, Oregon Health \& Science University, Portland, Oregon; ${ }^{\mathrm{c} K n i g h t}$ Cancer Institute, Oregon Health \& Science University, Portland, Oregon; 'Biostatistics Shared Resources, Knight Cancer Institute, Oregon Health \& Science University, Portland, Oregon; eOregon Health \& Science University - Portland State University School of Public Health, Portland, Oregon; fVancouver Prostate Centre, Department of Urologic Sciences, University of British Columbia, British Columbia, Canada; and 9Helen Diller Family

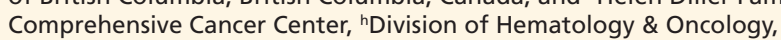
'Department of Epidemiology and Biostatistics, and 'Departments of Radiation Oncology and Urology, University of California San Francisco, San Francisco, California.

Submitted November 15, 2017; accepted for publication February 27, 2018.

Dr. Wyatt has disclosed that he has a consulting or advisory role with Sanofi. Dr. Youngren has disclosed that he receives research funding from Zenith Epigenetics. Dr. Beer has disclosed that he has stock or other ownership interests with Salarius Pharmaceuticals; has a consulting or advisory role with Abbvie, AstraZeneca, Churchill Pharmaceuticals, Clovis Oncology, Dendreon, Janssen Biotech, Astellas, Bayer, Boehringer Ingelheim, Janssen Oncology, Janssen Research \& Development, Johnson \& Johnson, Janssen Japan, F. Hoffmann La Roche, Merck, and Pfizer; and receives grant or research support from Astellas, Boehringer Ingelheim, Bristol-Myers Squibb, Dendreon, Janssen Research \& Development, Medivation, OncoGenex, Sotio, and Theraclone Sciences. Dr. Feng has disclosed that he has a leadership role at PFS Genomics; has served as a consultant for Clovis, Bayer, Medivation/Astellas, GenomeDx, Celgene, Dendreon, EMD Serono, Janssen Oncology, Ferring, and sanofi-aventis; receives grant or research support from Medivation/Astellas, Varian Medical Systems, and Celgene; and helped develop a molecular signature to predict radiation resistance in breast cancer, which was patented by the University of Michigan, and is in the process of being licensed to PFS Genomics. Dr. Small has disclosed that he has stock or other ownership interests with Fortis Therapeutics and Harpoon Therapeutics; receives honoraria from Janssen-Cilag; has a consulting and advisory role with Fortis Therapeutics, Gilead Sciences, and Valeant Pharmaceuticals International; and receives grant or research support from Janssen. Dr. Alumkal has disclosed that he has a consulting or advisory role with Astellas Pharma, Bayer, and Janssen Biotech; and that he receives research funding from Aragon, Astellas, Gilead Sciences, Novartis, and Zenith. The remaining authors have disclosed that they have no financial interests, arrangements, affiliations, or commercial interests with the manufacturers of any products discussed in this article or their competitors.

Correspondence: Joshi J. Alumkal, MD, Department of Medicine, Oregon Health \& Science University, 3303 SW Bond Avenue, CH14R, Portland, OR 97239. Email: alumkalj@ohsu.edu 
Lu et al

\section{Background}

Prostate cancer is the most common cancer and second leading cause of cancer-related death in men in the United States. ${ }^{1}$ Despite progress in recent years with 5 new drugs approved for the treatment of metastatic castration-resistant prostate cancer (CRPC), this lethal form of the disease remains incurable. ${ }^{2}$

We now know that CRPC is heterogeneous with distinct molecular subsets. ${ }^{3}$ One subset is defined by recurrent mutations in DNA repair genes whose loss of function (LoF) may lead to sensitivity to platinum chemotherapy or PARP inhibitors-a concept known as synthetic lethality, best studied in the context of BRCA gene alterations. ${ }^{3-7}$ Subsequent preclinical studies identified several additional DNA repair genes whose $\mathrm{LoF}$ is associated with PARP inhibitor sensitivity. ${ }^{8}$ Several groups have completed clinical trials with PARP inhibitors that have shown clinical benefit in CRPC. ${ }^{9-11}$ However, it is unclear to many practicing clinicians which patients with which specific defects in DNA repair genes should be recommended for PARP inhibitor treatment.

In this case series, we describe our institutional experience using single-agent PARP inhibitor therapy to treat patients with CRPC with DNA repair gene alterations. Specifically, we examined how tumor response and time on PARP inhibitor treatment differ depending on the gene mutations identified.

\section{Patients and Methods}

\section{Patients and Treatment}

Oregon Health \& Science University (OHSU) Institutional Review Board approval was obtained to retrospectively identify patients found to have a DNA repair gene alteration that would suggest sensitivity to PARP inhibitors in germline testing or testing of a metastatic CRPC sample. Patients who underwent biopsy had previously consented to participate on the West Coast Prostate Cancer Dream Team biopsy protocol. PARP inhibitor drug prescribing, follow-up management, dose modifications for toxicity, and discontinuation of therapy for clinical or radiographic progression was at the provider's discretion.

\section{Outcomes and Assessment}

Several metrics of antitumor activity were examined, including maximal decline in prostate-specific antigen (PSA) level while on therapy, radiographic response while on therapy, and duration of treatment measured from the start date of PARP inhibitor to the date of its discontinuation.

\section{Germline and Somatic Mutation Testing}

All subjects included in this case series had undergone germline testing and/or somatic mutational testing for DNA gene repair alterations prior to treatment with a PARP inhibitor. Four patients underwent germline testing (patients 1-4). Samples were obtained via buccal wash or peripheral blood mononuclear cell testing. Material was then sent either to the commercial Myriad Genetic Laboratories or the OHSU clinical laboratory for testing. Seven patients underwent somatic testing, performed in accordance with standard operating procedures (patients $1-3,5-8) .{ }^{12}$ Of these patients, 1 had tumor testing for BRCA2 alone (patient 1), 1 had circulating tumor DNA (ctDNA) testing for BRCA2 alone (patient 3), and 5 had tumor testing and genotyping with the Knight Diagnostic Laboratories' GeneTrails Comprehensive Solid Tumor Panel of 124 genes (patients 2, 5-8) (supplemental eTable 1, available with this article at JNCCN.org). Retrospectively, we used the PolyPhen algorithm to make functional predictions about the missense mutations identified in patients treated with PARP inhibitors in this series. ${ }^{13}$

\section{Results}

\section{Patients Examined}

Between June 2014 and June 2017, 8 patients with CRPC in OHSU clinics were treated with PARP inhibitors based on mutations in DNA repair genes. Baseline characteristics are summarized in Table 1. Follow-up data were collected through October, 2017. Patient 1 was treated with talazoparib and patients 2 through 7 were treated with olaparib. Prior to PARP inhibitor therapy, all patients had experienced disease progression on at least one regimen previously approved for the treatment of CRPC. None of these patients previously received platinum-based chemotherapy.

\section{Defects in DNA Damage-Repair Genes}

Figure 1 shows the alterations in DNA damage repair genes identified. Specific mutations are detailed in supplemental eTable 2. Copy number loss and stopgain mutations were identified in several patients. 
PARP Inhibitor Response in CRPC

\begin{tabular}{|c|c|}
\hline Characteristic & \\
\hline \multicolumn{2}{|l|}{ Age, y } \\
\hline Median & 68.5 \\
\hline Range & $52-73$ \\
\hline \multicolumn{2}{|l|}{ Total Gleason score } \\
\hline 6 & $1(12.5 \%)$ \\
\hline 7 & $0(0 \%)$ \\
\hline 8 & $2(25.0 \%)$ \\
\hline 9 & $3(37.5 \%)$ \\
\hline 10 & $0(0 \%)$ \\
\hline Unavailable & $2(25.0 \%)$ \\
\hline \multicolumn{2}{|c|}{ ECOG performance status score } \\
\hline 0 & $3(37.5 \%)$ \\
\hline 1 & $3(37.5 \%)$ \\
\hline 2 & $2(25.0 \%)$ \\
\hline $3-4$ & $0(0 \%)$ \\
\hline \multicolumn{2}{|l|}{ PSA, ng/mL } \\
\hline Median & 28.25 \\
\hline Range & $5.76-700.29$ \\
\hline \multicolumn{2}{|c|}{ Regimens for CRPC received prior to PARP inhibitor therapy, $\mathrm{n}$} \\
\hline 1 & $1(12.5 \%)$ \\
\hline 3 & $3(37.5 \%)$ \\
\hline$\geq 4$ & $4(50.0 \%)$ \\
\hline \multicolumn{2}{|c|}{$\begin{array}{l}\text { Life-extending therapies for metastatic CRPC received prior to } \\
\text { PARP inhibitor therapy, } \mathrm{n}\end{array}$} \\
\hline Enzalutamide & $7(87.5 \%)$ \\
\hline Abiraterone acetate & $4(50.0 \%)$ \\
\hline Docetaxel & $3(37.5 \%)$ \\
\hline Radium-223 & $1(12.5 \%)$ \\
\hline Sipuleucel-T & $1(12.5 \%)$ \\
\hline
\end{tabular}

Abbreviations: CRPC, castration-resistant prostate cancer; PSA, prostate-specific antigen.

In patients 1 and 3, this resulted in biallelic $\mathrm{LoF}$ in BRCA2. Patient 4 had a known germline monoallelic stop-gain mutation in BRCA2 but unknown status of the other allele.

A number of missense mutations in DNA damage-repair genes were also identified. Of note, none of these variants had previously been documented as pathogenic alleles. Patient 2's tumor had BRCA2 and FANCA mutations that were both of unknown significance. Patient 5's tumor harbored a FANCC mutation of unknown significance. Patient 6 had ATM copy number loss and an ATM mutation of unknown significance. Patient 7 had missense mutations in FAM175A, MLH3, and FANCA, all of un-

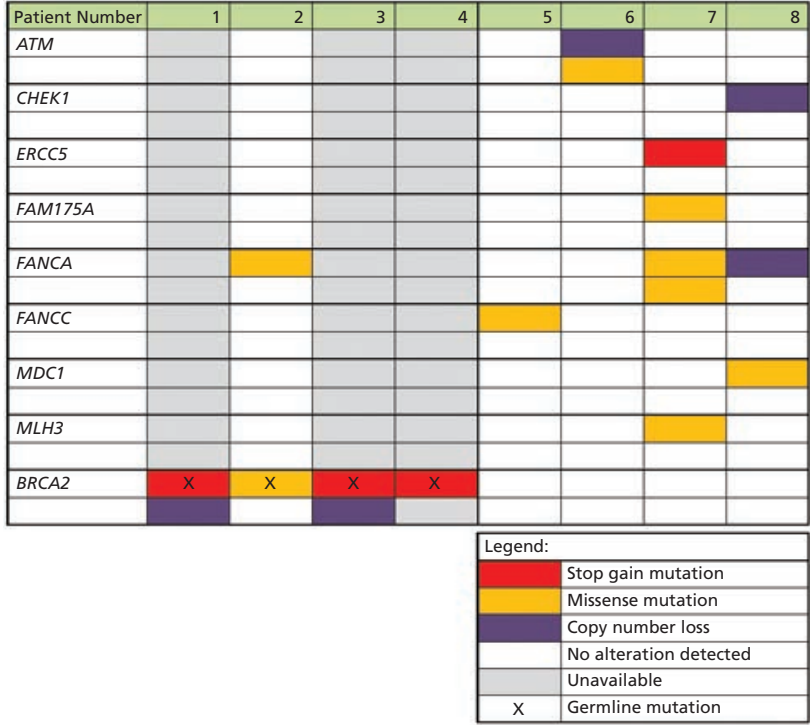

Figure 1. Genomic aberrations identified in the 8 patients found to have either germline and/or somatic alterations in DNA repair genes.

known clinical significance. Patient 8 had a missense mutation in MDC1 of unknown significance.

\section{Antitumor Activity}

PSA results were examined following PARP inhibitor treatment. PSA responses ( $>50 \%$ decline) were observed in 4 of 8 patients, all of whom had mutations in BRCA2. PSA level declines in these patients ranged from $64 \%$ to $100 \%$. Two of the responders had known biallelic LoF in BRCA2 (patients 1 and 3). One responder had a known LoF in one allele of BRCA2 but unknown status in the other allele (patient 4). The last responder (patient 2) had a germline missense mutation in BRCA2 and a somatic missense mutation in FANCA, both of unknown clinical significance (Figure 2). There was a significant difference in response in patients with BRCA2 gene alterations versus those with alterations in other DNA repair genes (Fisher exact test, 2 -sided $P=.03$ ); however, it is worth noting that patient 2 also carried a FANCA missense mutation that could also be responsible for driving the response to PARP inhibition. Of the 4 patients who had PSA responses, 3 underwent serial radiographic imaging and all 3 had radiographic responses by CT. The other subject (patient 4) had a 100\% decline in PSA level with a nadir of 0 , and thus did not undergo radiologic imaging due to the complete PSA response. 


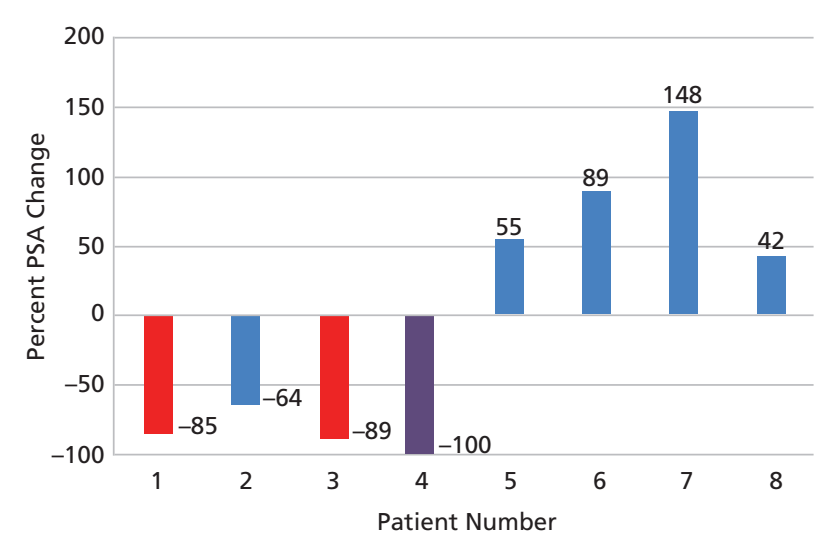

Figure 2. Waterfall plot for percent PSA change after PARP inhibitor treatment. Patients with known biallelic loss-of-function (LoF) DNA repair mutations are shown in red. Patients without known biallelic LoF mutations are shown in blue. The patient indicated in purple had germline testing only that showed a single LoF DNA repair mutation.

Median duration on treatment was 31.4 weeks (range, 20.9-78.3 weeks) for the 4 patients with BRCA2 alterations compared with 6.4 weeks (range, 2.4-13.0 weeks) in the 4 patients with non-BRCA2 DNA repair gene alterations (Wilcoxon rank sum test, $P=.03$ ) (Figure 3). At the time of writing, 1 patient (patient 4 who has a germline BRCA2 mutation) is still on PARP inhibitor therapy with a continued complete PSA response; 3 patients experienced disease progression and are still alive, and 4 others experienced disease progression and are now deceased.

\section{Discussion}

In this case series, we report our experience with singleagent PARP inhibitor therapy in patients with metastatic CRPC with DNA repair defects. In the largest series to date with PARP inhibitor therapy and somatic or germline mutational testing (TOPARP-A), 10 of 16 responders had biallelic mutations in homologous recombination DNA repair genes. ${ }^{11}$ Similarly, in our series, 2 of the 4 responders (patients 1 and 3 ) had confirmed biallelic LoF alterations in the homologous recombination DNA repair gene BRCA2. A third patient (patient 4) had known LoF in BRCA2 of one allele but unknown status of the other allele (Figure 1).

Interestingly, 1 of these 4 patients (patient 2) appeared to respond without biallelic loss in a DNA repair gene in the tumor specimen examined. This patient was started on therapy because of 2 separate monoallelic missense mutations. Subsequent analyses demonstrated that his FANCA p.E938Q muta- tion was rated as probably damaging by PolyPhen, and that his germline BRCA2 p.L2217V mutation was rated as possibly damaging by PolyPhen. Thus, we cannot conclude for certain which of these alterations was most critical for response. ${ }^{13}$ Response to PARP inhibition with only monoallelic LoF in a DNA repair gene has previously been noted in the TOPARP-A cohort. ${ }^{11}$ However, given the limited sample sizes of both this case series and the TOPARP-A cohort, data remain insufficient to guide PARP inhibitor therapy in patients whose tumors only harbor one LoF event in a DNA repair gene. ${ }^{11}$ There are also other explanations for this subject's response, including epigenetic mechanisms (ie, DNA methylation) leading to LoF of the second allele of FANCA or BRCA2; biallelic inactivation of other DNA repair genes not tested in this panel (supplemental eTable 1); or biallelic loss of BRCA2, FANCA, or other DNA repair genes in other tumor lesions not biopsied within this same patient.

Of the 4 patients who did not experience a response, several different DNA repair gene alterations were present in their tumors, including a number of monoallelic mutations in patients 5 (FANCC), 7 (FANCA, ERCC5, FAM175A, MLH), and 8 (CHEK1, FANCA, MDC1) (Figure 1, supplemental eTable 2). The lack of response seen in these 3 patients without clearly pathogenic biallelic LoF mutations is consistent with what was observed in TOPARP-A. ${ }^{11}$ One outlier was patient 6 , whose tumor biopsy harbored both an ATM copy number loss and an ATM p.R2459C missense mutation that was

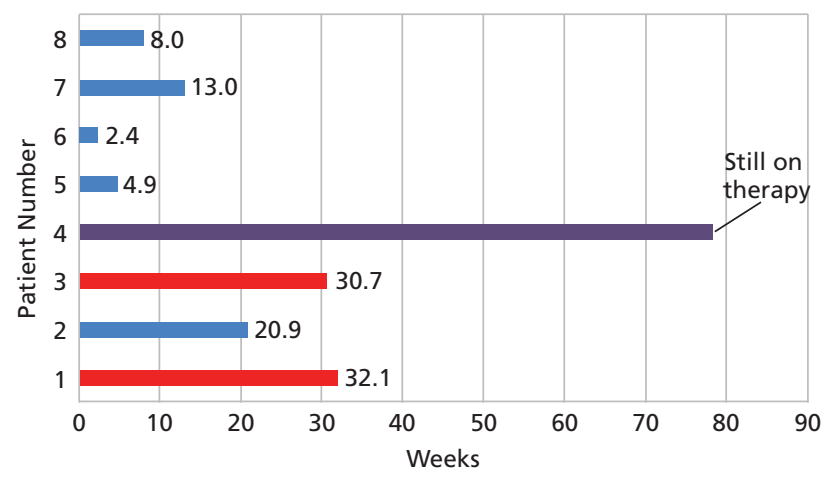

Figure 3. Swimmer's plot depicting time on PARP inhibitor treatment in weeks for 8 patients treated with PARP inhibitor therapy. Patients with known biallelic loss of function (LoF) DNA repair mutations are shown in red. Patients without known biallelic LoF mutations are shown in blue. The patient indicated in purple had germline testing only that showed a single LoF DNA repair mutation. Patient 4 is currently still on therapy 78 weeks after initiating PARP inhibitor treatment. 
predicted to be pathogenic by PolyPhen. Although the homozygous LoF alterations in ATM would have been considered "biomarker positive" in the TOPARP-A study and were predicted to lead to response to PARP inhibition, 2 of the nonresponders in that trial in fact had biallelic LoF mutations (including 1 patient with biallelic LoF ATM much like our patient). Thus, our data are consistent with TOPARP-A and suggest that additional tumor-intrinsic or host factors contribute to PARP inhibitor response. With the exception of patient 6 , responses were not observed in the other patients without known biallelic LoF events in DNA repair genes. These findings are consistent with what was observed in TOPARP-A and should serve as a cautionary note on treatment selection of patients for PARP inhibitor therapy who do not have confirmed functionally significant DNA repair gene mutations. . $^{811,14}$

This case series had several limitations. First, the sample size was small, and only one patient (patient 2) had both germline and tumor testing. In addition, we observed that patients harboring BRCA2 alterations had a significantly better response by multiple measures. However, whether patient 2's germline BRCA2 p.L3317V mutation was truly pathogenic and responsible for this patient's response remains unclear. Furthermore, on rare occasions, somatic mutation testing via GeneTrails can miss exon-level deletions, and we did not examine epigenetic mechanisms of gene silencing. Thus, we cannot conclude with certainty that patients classified as DNA repair gene alteration-negative (patients 5-8) did not in fact have exon-level deletions or epigenetic mechanisms of gene silencing.

\section{Conclusions}

A key question for the field moving forward is how best to stratify patients with CRPC to clinical trials or to treatment with off-label use of FDA-approved PARP inhibitors. ${ }^{14}$ Prospective clinical trials that are not stratified by specific DNA repair gene alterations, but that include mutational testing on all subjects, may help to answer this question. Moreover, incorporation of functional studies to confirm that variants of unknown significance are actually pathogenic will also be important. Finally, our and others' recent work demonstrates that ctDNA sequencing provides similar information as sequencing from a metastatic biopsy and that ctDNA sequencing may be a useful method to detect reversion mutations in BRCA2 at the time of PARP inhibitor resistance. ${ }^{15-18}$ Thus, ctDNA measurements may be an attractive approach to identify patients whose tumors harbor DNA repair gene mutations that lead to LoF and PARP inhibitor sensitivity.

\section{References}

1. Siegel RL, Miller KD, Jemal A. Cancer statistics, 2018. CA Cancer J Clin 2018;68:7-30.

2. Lorente D, Mateo J, Perez-Lopez R, et al. Sequencing of agents in castration-resistant prostate cancer. Lancet Oncol 2015;16:e279-292.

3. Robinson D, Van Allen EM, Wu YM, et al. Integrative clinical genomics of advanced prostate cancer. Cell 2015;161:1215-1228.

4. Mateo J, Boysen G, Barbieri CE, et al. DNA repair in prostate cancer: biology and clinical implications. Eur Urol 2017;71:417-425.

5. Cancer Genome Atlas Research Network. The molecular taxonomy of primary prostate cancer. Cell 2015;163:1011-1025.

6. Farmer H, McCabe N, Lord CJ, et al. Targeting the DNA repair defect in BRCA mutant cells as a therapeutic strategy. Nature 2005;434:917-921.

7. Castro E, Mateo J, Olmos D, et al. Targeting DNA repair: the role of PARP inhibition in the treatment of castration-resistant prostate cancer. Cancer J 2016;22:353-356

8. McCabe N, Turner NC, Lord CJ, et al. Deficiency in the repair of DNA damage by homologous recombination and sensitivity to poly(ADP-ribose) polymerase inhibition. Cancer Res 2006;66:8109-8115.

9. Hussain M, Carducci MA, Slovin S, et al. Targeting DNA repair with combination veliparib (ABT-888) and temozolomide in patients with metastatic castration-resistant prostate cancer. Invest New Drugs 2014;32:904-912.

10. Hussain M, Daignault $S$, Twardowski $P$, et al. Targeting androgen receptor and DNA repair in metastatic castration-resistant prostate cancer: results from NCI 9012. J Clin Oncol 2018;36:991-999.

11. Mateo J, Carreira S, Sandhu S, et al. DNA-repair defects and olaparib in metastatic prostate cancer. N Engl J Med 2015;373:1697-1708.

12. Tao DL, Bailey S, Beer TM, et al. Molecular testing in patients with castration-resistant prostate cancer and its impact on clinical decision making [published online June 28, 2017]. JCO Precis Oncol, doi: 10.1200/ PO.16.00067

13. Liu $X, W u$ C, Li C, et al. dbNSFP v3.0: A one-stop database of functional predictions and annotations for human nonsynonymous and splice-site SNVs. Hum Mutat 2016;37:235-241.

14. Michels J, Vitale I, Saparbaev M, et al. Predictive biomarkers for cancer therapy with PARP inhibitors. Oncogene 2014;33:3894-3907.

15. Quigley D, Alumkal JJ, Wyatt AW, et al. Analysis of circulating cell-free DNA identifies multiclonal heterogeneity of BRCA2 reversion mutations associated with resistance to PARP inhibitors. Cancer Discov 2017; 7:999_ 1005 .

16. Christie EL, Fereday S, Doig K, et al. Reversion of BRCA1/2 germline mutations detected in circulating tumor DNA from patients with highgrade serous ovarian cancer. J Clin Oncol 2017;35:1274-1280.

17. Wyatt AW, Annala M, Aggarwal R, et al. Concordance of circulating tumor DNA and matched metastatic tissue biopsy in prostate cancer [published online December 1, 2017]. J Natl Cancer Inst, doi: 10.1093/ jnci/djx118

18. Goodall J, Mateo J, Yuan W, et al. Circulating cell-free DNA to guide prostate cancer treatment with PARP inhibition. Cancer Discov 2017;7:1006-1017.

\section{See JNCCN.org for supplemental online content.}

